\title{
微任务驱动法在中职 CAD 教学中的实验研究
}

\section{Experimental Study on Micro Task Driven Method in CAD Teaching in Secondary Vocational School}

再超

Chao Ran

\author{
龙里县中等职业学校 中国·贵州 龙里 551200 \\ Longli Secondary Vocational School, Longli, Guizhou, 551200, China
}

\begin{abstract}
摘 要: 中等职业技术教学具有自己的特点, 同时 CAD 实验教学作为其中比较重视实际操作的一门课程, 具有一定的教 学难度。微任务驱动教学法具有一定的趣味性, 同时能够提升学生的自学能力, 在很多学科中获得了应用。论文研究了微 任务驱动教学法在中等职业技术教育 CAD 教学中的应用，希望提出的教学措施能够给相关专业的教育工作者带来启发。

Abstract: Secondary vocational and technical teaching has its own characteristics, and CAD experimental teaching, as a course which attaches more importance to practical operation, has certain teaching difficulty. Micro task driven teaching method is interesting and can improve students' self-learning ability. It has been applied in many subjects. This paper studies the application of micro task driven teaching method in CAD teaching of secondary vocational and technical education, hoping that the proposed teaching measures can bring inspiration to relevant professional educators.
\end{abstract}

关键词：教学法; 中等职业技术教育; $\mathrm{CAD}$ 实验教学

Keywords : teaching method; secondary vocational and technical education; CAD experiment teaching

DOI : $10.36012 /$ sde.v2i11.2374

\section{1 引言}

中等职业技术教育是中国教育体系的重要组成部分，在 教育体系中占有重要的地位。中等职业技术教育有效吸纳了 很多社会闲散人员，并且教会他们在社会进行生存的必要本 领，有效减轻了社会就业压力，并且促进了社会就业水平的 提高，同时也促进了社会生产水平的提升。中等职业技术教 育具有自己的特点, 一般来说, 选拔入学的学生知识功底比 较薄弱, 知识面不够宽广, 同时理论知识有欠缺, 但他们的 动手能力比较强, 敢于进行社会实践, 这又是他们不同于普 通大学学生的特点。因此, 如何进行中等职业技术教育, 基 于学生的特点取长补短, 教导学生学会直接能拿来应用的本 领, 是一项具有一定挑战性的课题。

近年来, 兴起了一种微任务驱动的教学法, 并且在很 多教学领域取得了不错的应用效果, 如何将它引入中等职业 技术教育中，还很少有人探讨和研究。

\section{2 微任务驱动教学法概述}

微任务驱动教学法是指在教学过程中, 以任务为牵引, 就是把教学任务分解到每一个具体的任务中，把任务布置给 学生, 指导学生通过自学完成任务, 老师对任务完成情况进 行讲评，同时对于完成任务过程中遇到的困难进行指导和讲 解，最终促进学生掌握授课知识 ${ }^{[1]}$ 。这种教学法的特点，可 以概括为以下几方面。

\section{1 微任务驱动教学法具有一定的趣味性}

微任务驱动教学法具有一定的趣味性，是把具体的知 识分解成一个个的任务，鼓励学生去解决任务，在解决任务 的过程中学习和掌握知识, 就像打游戏升级一样, 学生比较 有成就感，因此学生会主动地去学习，并且会想尽办法去自 己解决任务，这样学生就会把主要精力投入学习任务中，从 而在解决任务的过程中学到知识。微任务驱动教学法尤其适 用于比较难和比较枯燥的学科教学中, 当老师面对这些知识

【作者简介】再超（1983 ），男，贵州都匀人，讲师，从事工程管理研究。 
的授课束手无策时, 可以尝试采用这种教学方法。

\section{2 微任务教学法能够很好地锻炼学生的自学能力}

微任务驱动教学法将具体的知识点分解之后以做任务 的形式向学生展示, 鼓励学生自己动手查资料或者做实验将 这些任务解决, 这有助于锻炼学生的自学能力。学生想要解 决这些问题, 完成好任务, 必须要自己动手去查找资料, 在 查找资料的过程中, 就会掌握这些知识点, 从而学到知识, 这样的过程有助于锻炼学生的自学能力。这样一来, 学生的 自学能力就会得到提高, 以后再遇到类似任务的时候, 就知 道怎么去解决，无形中提高了分析问题、解决问题的能力。

\section{3 微任务驱动教学法具有一定的目标指向性}

微任务驱动教学法有一定的目标指向性, 必须要按照 教学大纲规定的具体知识点进行任务的设计和场景的规划, 只有这样, 才能确保任务中涉及的知识点符合教学大纲的规 定和要求, 才能确保教学活动可控且在正规的轨道上。因 此, 这就要求授课老师提出的每一个任务, 必须符合教学大 纲的要求, 原则上是为了完成本学科的教学目标设计的, 而 不能有多余的任务或者不符合规定的任务。这在一定程度上 对老师的教学能力提出了考验, 要求老师必须熟知教学大纲 的要求, 并且需要具备将教学大纲进行分解的能力, 这样才 能确保设计的任务具有一定的目标指向性, 学生才能够有的 放矢地进行学习, 这样一学期下来才能够确保教学计划的完 成。同时，教师在组织微任务驱动教学法实施教学任务的过 程中, 还需要关注学生的思想动态变化, 关注学生情感的变 化和价值观层次的培养, 确保学生始终以愉悦的身心投入到 任务解决的过程中，才能收到比较好的教学效果。

\section{3 微任务驱动教学法在 CAD 实验教学中的 应用措施}

中等职业技术教学任务中, $\mathrm{CAD}$ 实验教学直接面对工 程应用实际，具有一定的难度，同时更多地强调上机实习， 需要学生通过反复实践和练习才能学到知识, 提高能力 ${ }^{[2]}$ 。 因此, 在 $\mathrm{CAD}$ 实验教学任务中, 可以引入微任务驱动教学法, 通过这种新的教学方法促进教学任务的落实, 从而提升学生 的学习能力。

\section{1 分析学生的学习需求}

微任务驱动教学法最主要的特点, 就是将课堂的抽象的 知识点设计成一个个形象可观的任务, 引导学生去解决这些
任务，在解决任务的过程中提升能力。在采用微任务驱动教 学法进行教学的过程中, 需要全面掌握学生的需求, 这样才 能有的放矢地进行任务分解，才能确保分解的任务能够调动 学生学习的兴趣，才能够达到应有的教学效果。CAD 实验 教学是中等职业技术教育建筑专业的一本基础必修课，决定 了学生将来工作的基础, 只有具备了最基本的 CAD 制图能 力, 才能够胜任建筑工程的要求, 因此, 学生在进行 CAD 实验教学的学习过程中, 最基本的需求就是掌握制图能力和 结合工程实际的绘图技巧，教师在进行任务分解时，一定要 把握好这一点。

\section{2 做好微任务驱动教学过程中的任务设计和分解}

在利用微任务驱动教学法开展教学活动的过程中, 需 要结合具体的知识点, 以及学生的学习需求进行教学任务的 设计和分解。在设计和分解过程中, 需要注意面和点的结合, 具体来说就是要有一个总任务进行引领, 向学生讲明白到底 需要通过一个个具体的任务学到什么知识, 可以先向学生展 示一些实物或者成熟的作品，让学生提前进行思考，预想自 己想要达到的学习效果 ; 还可以将具体的教学任务分解成一 个个具体的点, 让学生围绕这些点开展工作, 这样能够有效 促进教学任务的完成，同时学生之间也可以锻炼协作和沟通 能力，这些对他们将来的发展都是有利的。

\section{4 结语}

总之, 如何利用先进和新颖的教学法, 提高课堂教学 质量和效果，是每一名老师需要思考的问题。中等职业技术 教育有自己的特点, 更是需要采用先进的教学法开展教学实 践活动, 微任务驱动教学法就适应了这一新的要求, 因此, 在中等职业技术教育中获得比较广泛的应用。笔者基于这种 认识, 探索了微任务驱动教学法在中等职业技术教育 CAD 实验教学中的具体应用方法, 并分析了这些应用方法的可行 性，希望能够为类似课程的教学实践者提供理论上的指导和 借鉴。

\section{参考文献}

[1] 朱江洁. 任务驱动法在中职 CAD 教学中的实验研究 [J]. 课程 教育研究 : 学法教法研究 ,2015(4):186-187.

[2] 潘先荣. 基于项目导向、任务驱动教学法在中职“电子 CAD”课程中的实践探究 [J]. 现代盐化工 ,2018,45(3):127-128. 